\title{
Macrofauna edáfica em área de pastagem no município de Macaíba, $\mathbf{R N}^{1}$
}

\author{
Ane Cristine Fortes da Silva ${ }^{2}$, Camila Costa da Nóbrega ${ }^{3}$, Victor Hugo de Farias Guedes ${ }^{4}$, Luan \\ Henrique Barbosa de Araújo ${ }^{5}$, César Henrique Alves Borges ${ }^{6}$
}

\begin{abstract}
Resumo: As diferentes coberturas vegetais e práticas culturais podem agir sobre a população da macrofauna edáfica, principalmente devido à diferença na estrutura da serrapilheira, que tem função nutricional e habitat para esses organismos. Objetivou-se caracterizar a abundância, riqueza e a diversidade da macrofauna edáfica em uma área de pastagem no município de Macaíba, RN. Os organismos foram coletados com armadilhas do tipo Provid alocadas aleatoriamente na área, permanecendo por um período de 96 horas em campo. As amostras foram levadas ao Laboratório de Ecologia Florestal/UFRN, onde os organismos amostrados foram extraídos e armazenados numa solução de álcool etílico 70\%. Em seguida, procedeu-se a contagem e identificação dos organismos dos grandes grupos taxonômicos e funcionais em laboratório. Avaliou-se a abundância, riqueza e diversidade através dos Índices de Diversidade de Shannon e Uniformidade de Pielou. Foram amostrados 212 indivíduos da macrofauna edáfica, distribuídos em dez grupos taxonômicos. O índice de Diversidade de Shannon e Equitabilidade de Pielou na área de estudo foi 0,5. Este resultado comprova a dominância do grupo Hymenoptera sobre os demais grupos taxonômicos. Os grupos Hymenoptera e engenheiros-do-ecossistema mostram-se mais expressivos na amostragem realizada indicando uma baixa riqueza, diversidade e equitabilidade da macrofauna edáfica na área de estudo.
\end{abstract}

Palavras-chave: Biota do solo; engenheiros-do-ecossistema; macroartrópodes do solo.

\section{Soil macrofauna in pasture area in the municipality of Macaíba, RN}

\begin{abstract}
Different vegetation cover and agricultural practices may affect the soil macrofauna population, mainly due to the difference in litter structure, which has nutritional function and habitat for these organisms. The objective of this study was to characterize the abundance, richness and diversity of the edaphic macrofauna in an area of pasture in the municipality of Macaíba, RN. We collected the macrofauna organisms with Provid type traps allocated randomly in the area, remaining for a period of 96 hours in the field. We take the samples to the Forest Ecology Laboratory / UFRN, where we extracted and stored the sampled organisms in a solution of ethyl alcohol $70 \%$. Then, we counted and identified the macrofauna organisms from the large taxonomic and functional groups. The abundance, richness and diversity were evaluated through the Shannon Diversity Indexes and Pielou Uniformity. Total number of macrofauna individuals sampled was 212, distributed in ten taxonomic groups. The Shannon Diversity Index and Pielou Equitability in the study area was 0.5. This result confirms the dominance of the Hymenoptera group over the other taxonomic groups. Hymenoptera groups and ecosystem engineers are more expressive in the sampling performed indicating low richness, diversity and equitability of the edaphic macrofauna in the study area.
\end{abstract}

Keywords: Soil biota; ecosystem engeneer; soil macroartropds.

${ }^{1}$ Submetido em 30/04/2018 e aprovado em 03/07/2018

${ }^{2}$ Mestre em Ciências Florestais; Professora, Instituto Federal de Educação, Ciência e Tecnologia da Paraíba (IFPB), Princesa Isabel - Paraíba, CEP: 58755-000; E-mail: ane.silva@ifpb.edu.br

${ }^{3}$ Mestre em Ciências Florestais; Doutoranda, Universidade Federal da Paraíba (UFPB), Centro de Ciências Agrária, Programa de PósGraduação em Ciência do Solo, Areia - Paraíba, CEP: 58397-000; E-mail: camila_cnobrega@ hotmail.com

${ }^{4}$ Graduação em Agronomia; Mestrando, Universidade Estadual de São Paulo (UNESP), Programa de Pós-Graduação em Agronomia (Ciência do Solo), Jaboticabal - São Paulo, CEP: 14884-900; E-mail: victorhguedes1@ gmail.com

${ }^{5}$ Mestre em Ciências Florestais; Doutorando, Universidade Federal Rural de Pernambuco (UFRPE), Programa de Pós-Graduação em Ciências Florestais, Recife - Pernambuco, CEP: 52171-900; E-mail: araujo.lhb@gmail.com

${ }^{6}$ Mestre em Ciências Florestais; Doutorando, Universidade Federal Rural de Pernambuco (UFRPE), Programa de Pós-Graduação em Ciências Florestais, Recife - Pernambuco, CEP: 52171-900; E-mail: cesarhenrique27@ yahoo.com.br

Revista Agropecuária Técnica, Areia-PB, v. 39, n. 2, p. 151-157, 2018

DOI: https://doi.org/10.25066/agrotec.v39i2.39565 


\section{Introdução}

A macrofauna do solo assume importante papel na formação e manutenção dos processos ecossistêmicos, sobretudo na ciclagem de nutrientes. O retorno de nutrientes por meio da deposição e decomposição pelos organismos edáficos dos resíduos orgânicos compõem a via mais importante do ciclo biogeoquímico de nutrientes nos ecossistemas.

A decomposição é definida como um processo de degradação física e transformação bioquímica de moléculas orgânicas complexas em moléculas orgânicas e inorgânicas mais simples (Bot e Benites, 2005). Conforme os organismos do solo fragmentam e degradam a matéria orgânica, os nutrientes são liberados no solo nas formas que as plantas podem absorver, este processo denominase mineralização, reintegrando ao ciclo interno de nutrientes Faltou referência.

A macrofauna edáfica responde às diversas intervenções antrópicas praticadas no meio ambiente (Lavelle e Spain, 2001). As diferentes coberturas vegetais e práticas culturais podem agir sobre a população da macrofauna alterando a taxa de decomposição dos resíduos. Isto se deve principalmente à diferença na estrutura do material decomposto, que tem função nutricional e serve de habitat para esses organismos.

Como exemplo os macroinvertebrados sofrem modificações em ambientes transformados em áreas de pastagens. Essas transformações vão da mudança de habitat, passando pela criação de microclima e chegando até a diferença no fornecimento de alimento aos indivíduos (Rezende et al., 2017). Reforçando Barros (2003), que menciona que a cobertura sob o solo tem efeitos importantes sobre a biota edáfica, modulando o aparecimento de grupos taxonômicos que são capazes de colonizar o solo.

Pesquisas tem avançado principalmente no que refere a demonstrar a macrofauna edáfica como indicador da qualidade do solo (Araujo et al., 2009; Paudel et al., 2012; Rousseau et al., 2013; Santos et al., 2016) . Deste modo, a abundância e diversidade desses organismos, assim como a existência de determinados grupos em detrimento de outros, podem ser utilizadas como bioindicadores da qualidade ambiental (Chaussod, 1996; Paoletti, 1999; Barros et al., 2003).
Portanto, a reunião de informações sobre a macrofauna do solo em ambientes antropizados pode fornecer indicativos da qualidade ambiental e subsidiar o planejamento da recuperação de áreas, pois além de avaliar os impactos das transformações antrópicas, o estudo da macrofauna edáfica serve como monitoramento das modificações após a remediação destas. E por fim, estes estudos podem auxiliar os produtores no acompanhamento e realização de diagnósticos da densidade e diversidade da população em uma determinada área de pastagem, visando melhor otimização da produtividade da mesma (Rezende et al. 2017).

Neste contexto, o presente estudo objetivo-se caracterizar a abundância, riqueza e a diversidade da macrofauna edáfica em uma área de pastagem no município de Macaíba, RN.

\section{Metodologia}

O trabalho foi desenvolvido durante o período de mês de ano a mês de ano em área de experimentação florestal na Unidade Acadêmica Especializada em Ciências Agrárias (UAECIA), Universidade Federal do Rio Grande do Norte (UFRN), campus de Macaíba - RN; em um ambiente de pastagem plantada com área experimental de 0,5 hectares, dividida em dez parcelas e em desuso nos últimos dois anos.

O clima local é caracterizado como tropical chuvoso com temperaturas elevadas e estação chuvosa de março a julho. A precipitação média anual é de $1.070 \mathrm{~mm}$, tendo a estação seca duração de seis a sete meses. A temperatura e umidade relativa média anual é aproximadamente $27,1^{\circ} \mathrm{Ce}, 76 \%$ respectivamente (IDEMA, 2008).

Para a amostragem da macrofauna edáfica foi realizada uma coleta, utilizando armadilhas do tipo Provid constituídas por uma garrafa PET com capacidade de dois litros, contendo quatro aberturas com dimensões de 3,0 x 3,0 cm na altura de $20 \mathrm{~cm}$ da base, com $300 \mathrm{~mL}$ de solução de detergente neutro $10 \%$ e três gotas de formaldeído.

As armadilhas foram alocadas aleatoriamente na área, buscando apenas impedir a proximidade com formigueiros para evitar que o número de indivíduos fosse superestimado. Os bordos das quatro aberturas das armadilhas foram enterrados ao nível da superfície do solo, permanecendo por um período de 96 horas. Após, as amostras foram

Revista Agropecuária Técnica, Areia-PB, v. 39, n. 2, p. 151-157, 2018

DOI: https://doi.org/10.25066/agrotec.v39i2.39565 
levadas ao Laboratório de Ecologia Florestal/UAECIA/UFRN, onde os organismos amostrados em cada armadilha com mais de 10 mm de comprimento ou com diâmetro corporal superior a dois milímetros foram extraídos e armazenados numa solução de álcool etílico a 70\%. Em seguida, procedeu-se a contagem e identificação dos organismos dos grandes grupos taxonômicos em laboratório (Storer et al., 1986; Borror e Delong, 1988).

$\mathrm{Na}$ avaliação do comportamento ecológico da macrofauna determinou-se a quantidade total de indivíduos (abundância), a riqueza de grupos taxonômicos (S) e a diversidade utilizando o Índice de Diversidade de Shannon (H), definido por

$$
H=-\sum p i \log p i
$$

em que

$$
p i=\frac{n i}{N}
$$

ni $=$ densidade de cada grupo;

$\mathrm{N}=\sum$ da densidade de todos os grupos.

Esse índice aceita valores que pode variar entre 0 a 5, sendo que a redução dos valores é o efeito de dominância de grupos em detrimento de outros (Begon et al., 1996). Já o índice de Uniformidade de Pielou (e) foi definido por:

$$
e=\frac{H}{\log S}
$$

em que

$\mathrm{H}=$ índice de Shannon;

$\mathrm{S}$ = Número de espécies ou grupos;
A macrofauna edáfica na área de estudo também foi caracterizada em relação ao grupo funcional a que pertence tem: decompositores, engenheiros do solo, predadores, herbívoros e outros grupos (Tarrá et al., 2012). Para a abundância de indivíduos da macrofauna edáfica nas parcelas avaliadas, foram calculadas as seguintes estatísticas: média $\bar{x}$; variância $\left(\mathrm{s}^{2}\right)$; desvio-padrão (s); erro-padrão $\left(S_{x}\right)$; intervalo de confiança a 5\% de probabilidade (IC 5\%) e coeficiente de variação em percentagem (CV \%). Para determinar a suficiência amostral necessária na amostragem de indivíduos da macrofauna das cinco espécies mais abundantes do estudo, visando a uma estimativa da média com $30 \%$ de erro, a $95 \%$ de probabilidade, foi utilizada a fórmula para população infinita em função da variância:

em que:

$$
n=\frac{t^{2} s^{2}}{E^{2}}
$$

$\mathrm{n}=$ número de amostras;

$\mathrm{t}^{2}=$ valor tabelar de " $\mathrm{t}$ " a $5 \%$ de probabilidade;

$\mathrm{s}^{2}=$ estimativa da variância;

$\mathrm{E}^{2}=$ erro previamente especificado $(30 \%)$;

\section{Resultados e Discussão}

Foram amostrados 212 indivíduos da macrofauna edáfica, distribuídos em dez grupos taxonômicos. O grupo Hymenoptera apresentou maior abundância na área estudada. Na Figura 1, encontra-se a composição relativa dos grupos taxonômicos da macrofauna edáfica na área de estudo.

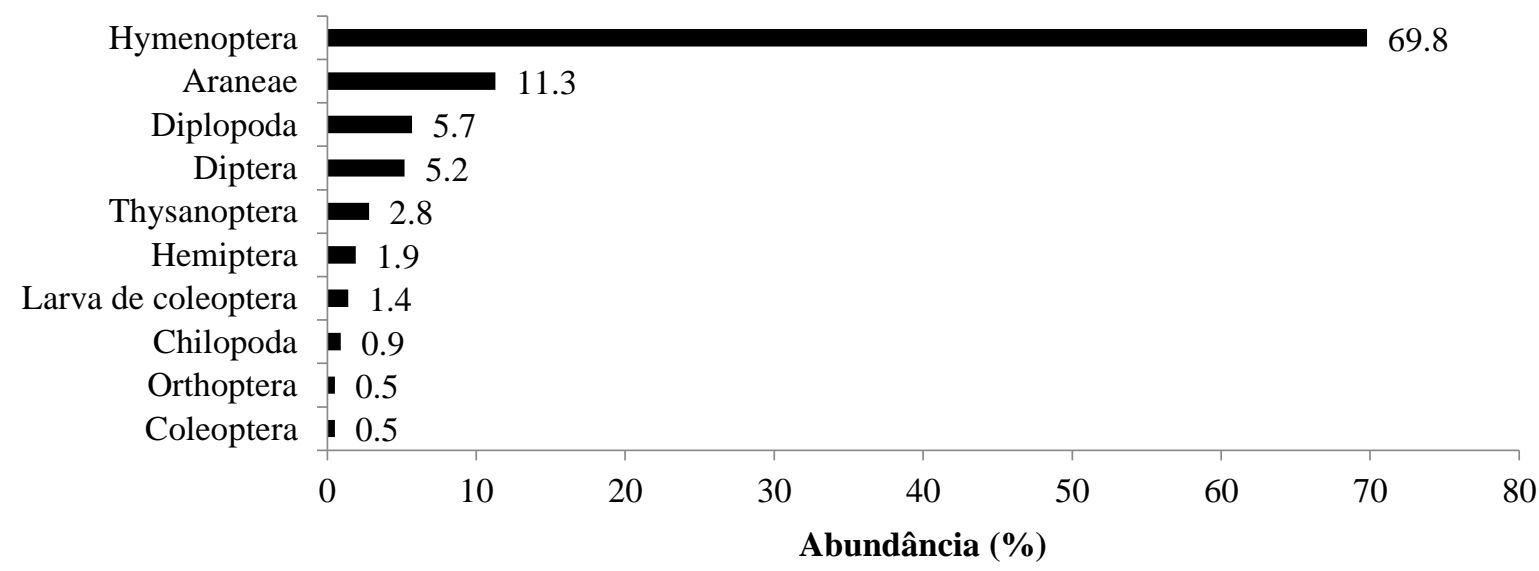

Figura 1 Abundância (\%) dos grupos taxonômicos da macrofauna edáfica na área de pastagem estudada.

Revista Agropecuária Técnica, Areia-PB, v. 39, n. 2, p. 151-157, 2018

DOI: https://doi.org/10.25066/agrotec.v39i2.39565 
A frequência relativa da abundância do grupo Hymenoptera é 2,3 vezes superior ao somatório das frequências dos demais grupos $(30,2 \%)$, indicando um desequilíbrio nas proporções de indivíduos de um mesmo grupo sobre os demais na comunidade avaliada. Além disto, verificou-se que este grupo é predominantemente composto por formigas. Isto pode estar relacionado ao fato de monoculturas fornecerem um único substrato alimentar favorecendo o desenvolvimento de determinados grupos faunísticos (Antoniolli et al., 2006, Baretta et al., 2003; Assad, 1997), como também a proximidade dos formigueiros ao experimento.

Rezende et al. (2017) constataram resultados semelhantes com predomínio de formigas em área de pastagem quando comparada a uma área queimada no Maranhão. Geremia et al. (2015) verificou resultado semelhante em pastagens submetidas a fonte de nutrientes orgânica e mineral.

A ordem Hymenoptera é um dos maiores grupos em número de espécies (Hanson e Gauld, 1995) e exibe fundamental importância para o funcionamento dos processos ecossistêmicos no solo. Além da fragmentação dos resíduos orgânicos, as formigas pertencentes a esse grupo realizam o revolvimento do solo com a formação de bioporos, uma das suas principais funções do solo. A partir da formação desses poros, tanto a infiltração da água quanto a aeração do solo é aumentada (Marques et al., 2014).

$\mathrm{O}$ segundo maior grupo encontrado neste estudo foi o Araneae com cerca de 24 indivíduos. A ordem Araneae corresponde a uma das maiores e mais diversificadas ordens de animais existentes. Indivíduos deste grupo possuem alta sensibilidade às mudanças de habitat, sendo potenciais como organismos indicadores do grau de conservação de um ambiente (Cardoso et al., 2004)

O índice de Diversidade de Shannon na área de estudo foi 0,5. Este resultado comprova a dominância do grupo Hymenoptera sobre os demais grupos taxonômicos encontrados na área. Resultados superiores foram constatados por Camargo et al. (2015) em área de pastagem no Pará e por Rezende et al. (2017). O Índice de Equitabilidade de Pielou foi 0,5. A riqueza encontrada na área estudada foi dez, correspondente aos dez grupos taxonômicos verificados no estudo.

Os baixos valores de diversidade de Shannon e equitabilidade de Pielou na área demonstram a desproporcionalidade entre os grupos edáficos no local, revelada pelo predomínio dos organismos da ordem Hymenoptera, o que está relacionado com a maior frequência encontrada. $\mathrm{O}$ conceito de diversidade descreve à variedade de organismos vivos, avaliando os aspectos da riqueza e equitabilidade, a menor igualdade entre grupos reduziu os índices de diversidade.

Em relação aos grupos funcionais, os organismos da macrofauna do solo observados neste estudo foram classificados em cinco grupos: decompositores, engenheiros-do-solo, predadores, herbívoros e outros grupos, seguindo a organização por grupo funcional modificada por Tarrá et al. (2012).

Os decompositores incluem os grupos taxonômicos Isopoda, Diplopoda, Diptera imaturos, Oligochaeta e Coleoptera imaturos. Os engenheiros-do-solo são compostos por Hymenoptera (Formicidae) e Isoptera. Já os predadores abrangem os organismos Araneae, Chilopoda, Dermaptera, Onicophora, Palpigradi, Phalangida e Pseudoscorpionida. Os herbívoros abarcam os grupos taxonômicos Thysanoptera, Hemiptera, Orthoptera, Homoptera adultos e imaturos. Já os outros grupos, abrangem organismos dos grupos Blattodea, Coleoptera, Hymenoptera (não Formicidae), Gastropoda, Neuroptera e Thysanoura.

Constata-se uma desproporcionalidade quanto aos grupos funcionais na área deste estudo (Figura 2), visto que o grupo engenheiros-do-solo compreende duas vezes mais o somatório das abundâncias dos demais grupos funcionais na área.

Verifica-se que os engenheiros-do-solo são os predominantes, isto porque a maior abundância foi de formigas com 147 indivíduos. Esse grupo apresenta maior impacto sobre a física do solo, transportando partículas e construindo galerias, estruturas e formando poros (Souza et al., 2015). 


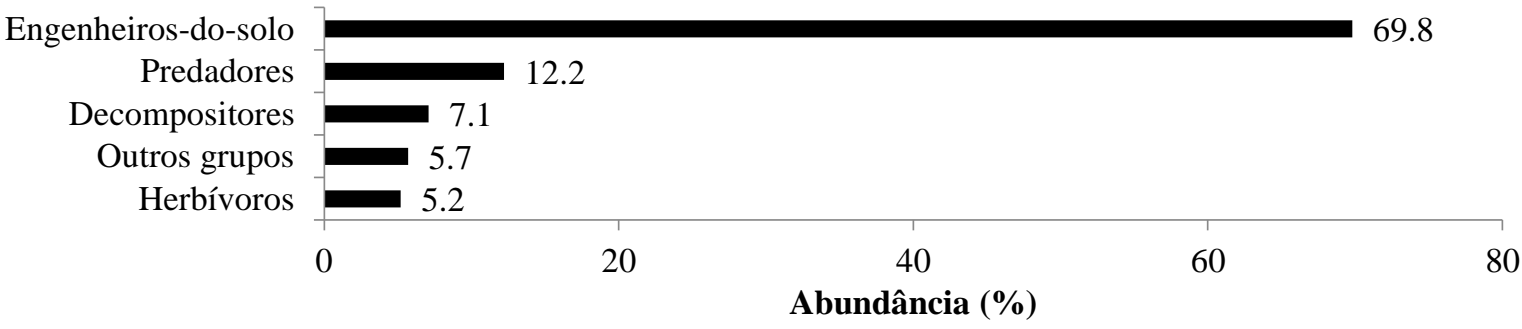

Figura 2 Abundância (\%) dos grupos funcionais da macrofauna edáfica na área de pastagem estudada.

Os predadores ocupam a segunda posição abrangendo cerca de $12,2 \%$ dos indivíduos, constituído pelos grupos taxonômicos Araneae e Chilopoda. Esses organismos atuam na regulação da cadeia trófica, controlando a população dos organismos, uma vez que estes se alimentam de outros organismos vivos (Souza et al., 2015).

Os decompositores, indivíduos imaturos de Coleóptera e pelos Diplopoda correspondeu a 15 indivíduos. Este grupo funcional caracteriza-se por serem transformadores da serapilheira, fragmentando os restos vegetais e disponibilizando para o acesso dos microorganismos do solo (Lavelle, 1997; Beck e Gasparotto, 2000).

Os herbívoros ou fitófagos correspondem aos organismos que consomem ou digerem tecidos vivos de vegetais. Neste estudo, incluíram-se neste grupo indivíduos de Orthoptera, Hemiptera e Thysanoptera, totalizando 5,2\% dos organismos.

Os indivíduos classificados em outros grupos não possuem classificação funcional predominante. Neste estudo incluíram indivíduos dos grupos taxonômicos Coleoptera e Diptera adultos, abrangendo $5,7 \%$ dos organismos verificados.

Os dados amostrados dos cinco grupos de maior abundância neste estudo foram analisados para determinar a intensidade amostral necessária na coleta de macrofauna do solo, visando a uma estimativa da média com $30 \%$ e $95 \%$ de probabilidade.

O menor coeficiente de variação e erro de amostragem relativo constatado neste estudo foi para a abundância dos indivíduos do grupo taxonômico Hymenoptera e o maior para Diplopoda (Tabela 1).

Tabela 1 Análise estatística das amostras de macrofauna edáfica na pastagem estudada

\begin{tabular}{lccccc}
\hline $\begin{array}{l}\text { Variáveis } \\
\text { estatísticas }\end{array}$ & Hymenoptera & Araneae & Diplopoda & Diptera (imaturo) & Thysanoptera \\
\hline Média & 24,67 & 4,00 & 2,00 & 1,83 & 1,83 \\
Variância & 34,67 & 3,20 & 7,20 & 2,97 & 2,97 \\
Desvio-padrão & 5,89 & 1,79 & 2,68 & 1,72 & 1,72 \\
Erro padrão & 2,40 & 0,73 & 1,10 & 0,70 & 0,70 \\
Erro de amostragem & 19,64 & 36,79 & 110,37 & 77,28 & 77,28 \\
relativo & {$[19,82-29,51]$} & {$[2,53-5,47]$} & {$[0,21-4,21]$} & {$[0,42-3,25]$} & {$[0,42-3,25]$} \\
IC $(\alpha=5 \%)$ & 23,87 & 44,72 & 134,16 & 93,95 & 93,95 \\
CV & 21 & 74 & 669 & 328 & 328 \\
n & 21 &
\end{tabular}

IC: intervalo de confiança; $\mathrm{CV}$ : coeficiente de variação; n: número de amostras suficientes.

De acordo com a análise estatística, o grupo Diplopoda exige um maior número de unidades amostrais para amostragem de seus indivíduos, enquanto que o grupo Hymenoptera necessita de um menor número de unidades amostrais. Isto pode estar relacionado à locomoção, alimentação e distribuição destes organismos no solo.
As formigas movimentam-se com maior velocidade e por esta razão conseguem estar distribuídos em maior área para buscar alimento, favorecendo sua amostragem. Já os Diplopoda possuem movimentos mais lentos e realiza a escavação do material orgânico para alimentação, o que favorecem sua localização pontual na área 
dificultando sua amostragem. Assim, é necessário um maior número de coletores para a amostragem desta ordem, assim como os organismos dos grupos taxonômicos Díptera (imaturo) e Thysanoptera.

\section{Conclusão}

O grupo Hymenoptera e engenheiros-doecossistema mostram-se mais expressivos na amostragem realizada indicando uma baixa riqueza, diversidade e equitabilidade da macrofauna edáfica na área de estudo.

Os cinco grupos taxonômicos mais abundantes na área de estudo revelam-se divergentes quanto à suficiência amostral, sendo necessário um maior número de unidades amostrais para uma melhor representatividade da população da macrofauna edáfica na área estudada.

\section{Referências}

Antoniolli, Z. I.; Conceição, P. C.; Böck, V.; Port, O.; Silva, R. F. Método alternativo para estudar a fauna do solo. Ciência Florestal, v.16, n.4, p.407-417,

2006.

http://dx.doi.org/10.5902/198050981922

Araújo, A. O.; Mendonça, L. A. R.; da Franca, R. M.; Feitosa, J. V.; de Moura Araújo, S. A., Simplício, A. A. F.; de Oliveira, J. F. Avaliação da densidade da macrofauna edáfica como indicador da degradação de solos submetidos a manejo florestal de vegetação nativa na Chapada do Araripe. Águas Subterrâneas, v.1, suplemento, $\quad$ p.1-12, 2009. https://aguassubterraneas.abas.org/asubterraneas/article/vi ew/21995

Assad, M. L. L. Fauna do solo. In: Vargas, M. A. T.; Hungria, M. A. T. Biologia dos solos dos Cerrados. Planaltina: EMBRAPA-CPAC, 1997, p. 363-443.

Baretta, D.; Mafra, A. L.; Santos, J. C. P.; Amarante, C. V. T. Bertol, I. Análise multivariada da fauna edáfica em diferentes sistemas de preparo e cultivo do solo. Pesquisa Agropecuária Brasileira, v.41, n.11, p.16751679 , 2006. https://seer.sct.embrapa.br/index.php/pab/article/view/733 1

Barros, E.; Neves, A.; Blanchart, E.; Fernandes, E. C.; Wandelli, E.; Lavelle, P. Development of the soil macrofauna community under silvopastoral and agrosilvicultural systems in Amazonia. Pedobiologia, v.47, n.3, p.273-280, 2003. https://doi.org/10.1078/0031-4056-00190

Beck, L.; Gasparotto, L. Soil fauna and litter decomposition in primary and secondary forest and a mixed culture system in Amazonia. Shift Project ENV 052. Final Report 1996-1999. Karlsruhe, 2000. 291p.

Begon, M.; Harper, J. L.; Townsend, C. R. Ecology: Individuals, Populations and Communities. 3. ed. Oxford: Blackwell Science, 1996. 945p.

Borror, D. J.; Delong, D. M. Introdução ao estudo dos Insetos, São Paulo: Ed. Edgard Blucher Ltda, 1988. 653p.

Bot, A.; Benites, J. The importance of soil organic matter: Key to drought-resistant soil and sustained food and production. FAO Soils Bulletin, 80. Roma: FAO, 2005. 80p.

Camargo, H. S.; Almeida, D. S.; Ferreira, L. O.; Silva, N. R.; Rodrigues, D. M. Diversidade de artrópodes em diferentes sistemas de manejo do solo na Amazônia Oriental. Cadernos de Agroecologia, v.10, n.3, p.1-5, 2015. http://revistas.abaagroecologia.org.br/index.php/cad/article/view/19159

Cardoso, P., Silva, I., De Oliveira, N. G.; Serrano, A. R. M. Higher taxa surrogates of spider (Araneae) diversity and their efficiency in conservation. Biological Conservation, v.177, n. $4, \quad$ p.453-459, 2004. https://doi.org/10.1016/j.biocon.2003.08.013

Chaussod, R. La qualité biologique des sols: evaluation et implications. Étude et Gestion des Sols, v.3, n.4, p.261-278, 1996. http://www.afes.fr/wpcontent/uploads/2017/10/EGS_3_4_CHAUSSOD.pdf

Geremia, E. V., Segat, J. C., Fachini, I. A., da Fonseca, E. O.; Baretta, D. Fauna edáfica em pastagem perene sob diferentes fontes de nutrientes. Scientia Agraria, v.16, n.4, p.17-30, 2015. http://dx.doi.org/10.5380/rsa.v16i4.47802

Hanson, P.E.; Gauld, I.D. (Eds.). The Hymenoptera of Costa Rica. Oxford: Oxford University Press, 1995. 893p.

Instituto de Desenvolvimento Sustentável e Meio Ambiente do Rio Grande do Norte - IDEMA. 2008. v 10. 35p. Disponível em: <http://www.idema.rn.gov.br/contentproducao/aplicacao/ 
idema/socio_economicos/arquivos/Perfil\%202008/Natal. pdf> Acesso em: 10 de Agosto de 2013.

Lavelle, P. Faunal activities and soil processes: adaptative strategies that determine ecosystem function. Advances Ecological Research, v.27, p.93-132, 1997. https://doi.org/10.1016/S0065$\underline{2504(08) 60007-0}$

Lavelle, P.; Spain, A.V. Soil ecology. Dordrecht: Kluwer Academic Pub., 2001. 654p.

Marques, D. M.; Silva, A. B.; Silva, L. M.; Moreira, E. A.; Pinto, G. S. Macrofauna edáfica em diferentes coberturas vegetais. Bioscience Journal, v.30, n.5, p.1588-1597, 2014. http://www.seer.ufu.br/index.php/biosciencejournal/articl e/view/22926

Paoletti, M. G. Using bioindicators based on biodiversity to assess landscape sustainability. Agriculture. Ecosystems and Environment, v.74, p.1-18, 1999. http://biobioindicator.org/information/Paoletti_1999_2.pdf

Paudel, B. R.; Udawatta R. P.; Kremer R. J.; Anderson S. H. Soil quality indicator responses to row crop, grazed pasture, and agrofores try buffer management. Agroforestry Systems, v.84, n.2, p.311-323, 2012. https://doi.org/10.1007/s10457-011-9454-8

Rezende, L. P.; de França Portela, G.; Macedo, N. C.; Diniz, K. D. Identificação da macrofauna do solo em pastagem de Panicum maximum jacq e área submetida à queimada no município de Sambaíba-MA. Biodiversidade, v.16, n.1, p.155-166,
https://www.gvaa.com.br/revista/index.php/RBGA/article /viewFile/6005/5088

Rousseau L.; Fonte S. J.; Téllez O.; van der Hoek R.; Lavelle P. Soil macrofauna as indicators of soil quality and land use impacts in small holder agroecosystems of western Nicaragua. Ecological Indicators, v.27, p.71-82, 2013. https://doi.org/10.1016/j.ecolind.2012.11.020

Santos, L. F.; Calixto, J. S.; Duarte, E. G.; Mota, L. P. L.; Pereira, R. T. G. Densidade de minhocas como indicador de qualidade do solo. Cadernos de Agroecologia, v.10, n.3, p.1-5, $2015 . \quad$ http://revistas.abaagroecologia.org.br/index.php/cad/article/download/1881 8/13209/

Souza, M. H.; Vieira, B. C. R.; Oliveira, A.P. G.; Amaral, A. A. Macrofauna do solo. Enciclopédia Biosfera, v.11, n.22, p.115-131, 2015.

http://www.conhecer.org.br/enciclop/2015E/Macrofauna. pdf

Storer, T. I.; Usinger, R. L.; Stebbins, R. C.; Nibakken, J. W. Zoologia Geral. $6^{a}$ ed. São Paulo: Editora Nacional. 1986. 816 p.

Tarrá, I. L.; Luizão, F. D. J.; Wandelli, E. V.; Teixeira, W. G.; Morais, W. J.; Fernandes, E. C.; Pérez, N. V. Grupos funcionais da macrofauna e macroporos do solo em sistemas agroflorestais da Amazônia Central. Prospectiva, v.10, n.1, p.6-17, 2012. http://dx.doi.org/10.15665/rp.v10i1.391 